\title{
INTERVALO HÍDRICO ÓTIMO E COMPACTAÇÃO DO SOLO COM CULTIVO CONSORCIADO DE MILHO E BRAQUIÁRIA $^{(1)}$
}

\author{
Juliano Carlos Calonego ${ }^{(2)}$, Emerson Borghi ${ }^{(3)}$ \& Carlos Alexandre \\ Costa Crusciol ${ }^{(4)}$
}

\begin{abstract}
RESUMO
O cultivo consorciado entre a espécie forrageira e a cultura produtora de grãos garante a produção de forragem no outono-inverno, além de palha para cobertura do solo em sistema semeadura direta. $O$ aporte de matéria seca radicular no perfil do solo possibilita a melhoria da qualidade estrutural do solo. Objetivouse com este experimento verificar as alterações nos atributos físicos e físicohídricos do solo com o cultivo de milho solteiro e consorciado com Brachiaria brizantha. O experimento foi conduzido na Fazenda Experimental Lageado (UNESP, Botucatu-SP), entre os anos agrícolas de 2002/2003 e 2003/2004. Foram coletadas amostras com estrutura preservada de solo, por meio de anéis volumétricos, nas camadas de 0 a 20 e 20 a $40 \mathrm{~cm}$ de profundidade, em trincheiras abertas em parcelas cultivadas com milho e mantidas em pousio na entressafra, bem como em parcelas cultivadas com milho consorciado com $B$. brizantha na linha de semeadura, mantendo a forrageira para pastagem após a colheita do milho. A partir dessas amostras, foi avaliado o Intervalo Hídrico Ótimo (IHO), por meio de curvas de resistência à penetração e de retenção de água, em amostras com diferentes densidades do solo. O cultivo solteiro de milho na safra de verão é suficiente para melhorar as características físicas e estruturais do solo na camada de 0 a $20 \mathrm{~cm}$. O cultivo de braquiária em consórcio com o milho por dois anos consecutivos melhora as condições físicas e estruturais do solo na camada de 20 a $40 \mathrm{~cm}$, com redução da resistência mecânica à penetração e aumento da densidade crítica do solo.
\end{abstract}

Termos de indexação: integração lavoura-pecuária, Zea mays, Brachiaria brizantha.

\footnotetext{
(1) Recebido para publicação em 21 de junho de 2010 e aprovado em 18 de agosto de 2011.

(2) Professor Doutor. Centro de Ciências Agrárias, UNOESTE. Rodovia Raposo Tavares, km 572. CEP 19067-175 Presidente Prudente (SP). E-mail: juliano@unoeste.br

(3) Pesquisador, Doutor, Empresa Brasileira de Pesquisa Agropecuária (EMBRAPA-CNPASA). Quadra 103 Sul, Av. JK, Lote 17, CEP 77015-012 Palmas (TO). E-mail: emerson.borghi@embrapa.br

(4) Professor Titular, Departamento de Produção Vegetal, Faculdade de Ciências Agronômicas, UNESP. Caixa Postal 237, CEP 18603-970 Botucatu (SP). E-mail: crusciol@fca.unesp.br
} 


\title{
SUMMARY: LEAST LIMITING WATER RANGE AND SOIL COMPACTATION AS RELATED TO INTERCROPPED MAIZE AND BRACHIARIA
}

\begin{abstract}
Intercropping of a forage and main grain crop ensures forage production in the autumnwinter, and straw for mulching in the no-tillage system. The contribution of dry root matter in the soil profile allows the improvement of soil structural quality. The objective of this research was to evaluate changes in soil physical and physico-hydric properties with the cultivation of maize grown alone and intercropped with Brachiaria brizantha. The study was carried out on the Experimental Farm Lageado (UNESP, Botucatu-SP) in the growing seasons 2002/2003 and 2003/2004. Undisturbed soil was sampled from the layers $0-20$ and $20-40 \mathrm{~cm}$ by volumetric rings in plots growing maize and left fallow between harvest and in plots intercropped with maize and B. brizantha in the row, where the grass was maintained for grazing after maize harvest. The least limiting water range (LLWR) of these samples was evaluated, based on curves of penetration resistance and water retention in soil samples with different bulk densities. Maize grown alone is sufficient to improve the physical and structural characteristics of the soil in the 0-20 cm layer. Brachiaria intercropped with maize for two years improves the soil physical and structural properties in the layer $20-40 \mathrm{~cm}$ as well, reducing penetration resistance and increasing bulk density.
\end{abstract}

Index terms: crop livestock systems, Zea mays, Brachiaria brizantha.

\section{INTRODUÇÃO}

No sistema de integração lavoura-pecuária (ILP), por meio da consorciação de duas gramíneas, a forrageira tem a função de fornecer alimento para a exploração pecuária, a partir do final do verão até início da primavera, e, posteriormente, de formação de palhada, para o cultivo da cultura produtora de grãos, em Sistema Semeadura Direta (SSD). Esse sistema pode vir a ser uma alternativa para o agricultor ou agropecuarista, visto que em muitas regiões do Brasil o cultivo de safrinha tem apresentado insucesso, em face da baixa disponibilidade hídrica e irregularidade na precipitação pluvial no período outono/inverno (Zanine et al., 2006).

Benefícios importantes têm sido observados na estruturação do solo em médio e longo prazo com a utilização de plantas de cobertura com alto potencial de fixação de carbono e que possuam sistema radicular volumoso e agressivo (Dias Júnior, 2000), com capacidade de crescer em solos com alta resistência à penetração, criando poros por onde as raízes da cultura subsequente possam crescer (Silva \& Rosolem, 2001; Calonego \& Rosolem, 2008). Segundo Andrade et al. (2009), as culturas de cobertura, especialmente as gramíneas, favoreceram a agregação do solo na camada superficial, mantendo-a ou aumentando-a em relação ao solo sob mata nativa. Estes autores concluíram também que o cultivo de milho consorciado com braquiária foi um dos que proporcionaram boa qualidade física ao solo.

Os resultados gerados com ILP mostram que a braquiária constitui opção de destaque entre as espécies com potencial para promover melhorias à estrutura do solo, em razão da quantidade, qualidade e distribuição de fitomassa radicular que adiciona ao solo (Kluthcouski et al., 2004; Fonseca et al., 2007; Denardini et al., 2008) e da satisfatória tolerância à compactação do solo (Silva, 2004), além de não interferir significativamente na produtividade do milho (Jakelaitis et al., 2005; Borghi \& Crusciol, 2007; Crusciol et al., 2009).

A avaliação física do solo é de difícil diagnóstico, razão pela qual Letey (1985) sugere que a avaliação da qualidade física do solo deva ser feita por meio de atributos físicos do solo relacionados com o crescimento das plantas, tendo a água como uma variável de equilíbrio, amenizando ou agravando os efeitos da aeração e da resistência à penetração.

Nesse sentido, Silva et al. (1994) desenvolveram o método do Intervalo Hídrico Ótimo (IHO) como ferramenta para avaliação física do solo. $\mathrm{Na}$ determinação do IHO assumem-se alguns valoreslimite para o ótimo crescimento das plantas (Silva et al., 1994), como porosidade de aeração superior a $10 \%$ (Grable \& Siemer, 1968), resistência à penetração das raízes inferior a $2 \mathrm{MPa}$ (Taylor, 1974) e quantidade de água no solo que esteja entre a capacidade de campo e o ponto de murcha permanente, ou seja, entre os potenciais de -0,01 e -1,5 MPa, respectivamente.

O IHO pode ter como limite superior o teor de água no solo na condição de capacidade de campo $(\theta C C)$ ou o teor de água em que a porosidade de aeração se encontra no valor de $10 \%(\theta \mathrm{PA})$. Como limite inferior, pode-se ter o teor de água do solo na condição de ponto de murcha permanente ( $\theta \mathrm{PMP}$ ) ou teor de água em que a resistência do solo à penetração seja igual a 2,0 MPa ( $(\theta \mathrm{RP})$ (Silva et al., 1994). 
Tendo em vista o exposto, o objetivo deste trabalho foi verificar possíveis alterações na resistência mecânica à penetração e no IHO do solo com o cultivo consorciado de milho e Brachiaria brizantha cv. Marandu, por meio da ILP em sistema de semeadura direta.

\section{MATERIAL E MÉTODOS}

O experimento foi realizado na Fazenda Experimental Lageado da FCA/Unesp, em Botucatu$\mathrm{SP}$, a $22^{\circ} 51^{\prime} \mathrm{S}$ e $48^{\circ} 26^{\prime} \mathrm{W}$, a $740 \mathrm{~m}$ de altitude, nos anos agrícolas de 2002/2003 e 2003/2004. O clima predominante na região é do tipo Cwa, segundo Köppen, ou seja, tropical de altitude, com inverno seco e verão quente e chuvoso (Lombardi Neto \& Drugowich, 1994). O solo da área experimental é um Nitossolo Vermelho (Embrapa, 2006) manejado em SSD desde o ano de 1997, utilizando a rotação de culturas milho/aveia-preta/soja/milho/milho/aveiapreta/soja/aveia-preta/milho/aveia-preta.

$\mathrm{O}$ delineamento experimental foi em blocos casualizados, em esquema fatorial $2 \times 2$, com quatro repetições. Os tratamentos constaram da repetição por dois anos (2002/2003 e 2003/2004) de cultivo de milho nos sistemas de produção envolvendo milho solteiro na safra de verão e pousio na entressafra (MS) e milho consorciado simultaneamente com Brachiaria brizantha cv. Marandu na linha de semeadura (MBL), mantendo pastagem na entressafra, fazendo-se comparações entre os sistemas nas camadas de 0-20 e 20-40 cm de profundidade.

Cada parcela foi constituída por 4,5 m de largura e $5 \mathrm{~m}$ de comprimento, com experimento ocupando uma área total de $450 \mathrm{~m}^{2}$. O híbrido de milho utilizado foi o AG 9010, e a espécie forrageira foi a Brachiaria brizantha Stapf cv. Marandu. Antes da instalação do experimento, setembro de 2002 , foram coletadas amostras de solo na camada de 0 a $0,20 \mathrm{~m}$ de profundidade, para análise de fertilidade (Raij et al., 2001) e granulométrica (Embrapa, 1997). Os resultados revelaram as seguintes características: $\mathrm{pH}$ $\left(\mathrm{CaCl}_{2}\right)$ de 4,8; $23 \mathrm{~g} \mathrm{~kg}^{-1}$ de MO; $19 \mathrm{mg} \mathrm{dm}^{-3}$ de P (resina); $1,8 \mathrm{mmol}_{\mathrm{c}} \mathrm{dm}^{-3}$ de K; $19 \mathrm{mmol}_{\mathrm{c}} \mathrm{dm}^{-3} \mathrm{de} \mathrm{Ca}$; $13 \mathrm{mmol}_{\mathrm{c}} \mathrm{dm}^{-3} \mathrm{de} \mathrm{Mg} ; 55 \mathrm{mmol}_{\mathrm{c}} \mathrm{dm}^{-3} \mathrm{de} \mathrm{H}+\mathrm{Al} ; 38 \%$ de $\mathrm{V}$, além de 490, 210 e $300 \mathrm{~g} \mathrm{~kg}^{-1}$ de argila, areia e silte, respectivamente. Realizou-se a calagem superficial, em 15/10/2002, sobre o resíduo vegetal remanescente na área, na dose de $2.500 \mathrm{~kg} \mathrm{ha}^{-1}$ de calcário dolomítico (PRNT $=90 \%)$, com o objetivo de elevar a saturação por bases a 70 \% (Raij et al., 1996).

As semeaduras do milho foram realizadas nos dias $13 / 12 / 2002$ e 13/12/2003, por meio de semeadoraadubadora para plantio direto, com três linhas espaçadas de 0,90 m, para obtenção de estande de 55.000 plantas ha $^{-1}$, sendo as semente depositadas a $5 \mathrm{~cm}$ de profundidade. No tratamento envolvendo o consórcio entre milho e braquiária, utilizou-se uma quantidade de sementes de forrageira equivalente a $10 \mathrm{~kg} \mathrm{ha}^{-1}(\mathrm{VC}=34 \%)$, sendo as sementes de braquiária misturadas ao adubo e acondicionadas no compartimento de fertilizante da semeadora, distribuídas na profundidade de $8 \mathrm{~cm}$, ou seja, abaixo da semente de milho, conforme Kluthcouski et al. (2000).

Para determinação do IHO conforme descrito em Silva et al. (1994), no segundo ano de condução do experimento, após a rebrota da braquiária decorrente de simulação de pastejo e dessecação desta para produção de cobertura morta, realizou-se no dia 20 de novembro de 2004 a coleta de amostras com estrutura preservada, por meio de anéis volumétricos de $0,05 \mathrm{~m}$ de altura por $0,05 \mathrm{~m}$ de diâmetro interno, em trincheira aberta com dimensões aproximadas de $0,30 \mathrm{~m}$ de largura, $0,60 \mathrm{~m}$ de comprimento e $0,50 \mathrm{~m}$ de profundidade, no centro de cada parcela. A amostragem foi realizada no centro das camadas de 0 a 0,20 e 0,20 a $0,40 \mathrm{~m}$, utilizando um suporte de aço para os anéis e marreta de $1 \mathrm{~kg}$; foram retiradas oito amostras por camada, em cada trincheira.

O IHO foi determinado calculando-se um valor de IHO para cada amostra, em função da densidade do solo. Os limites do IHO foram associados a valores críticos de umidade do solo quanto ao potencial mátrico, resistência do solo à penetração e porosidade de aeração, representados, respectivamente, pelo teor de água na capacidade de campo ( $\theta c c)$, ou seja, pelo teor de água retida no solo com potencial mátrico de $-0,01 \mathrm{MPa}$ (Haise et al., 1955); pelo teor de água no ponto de murcha permanente $\left(\theta_{\mathrm{PMP}}\right)$, ou seja, pelo teor de água retida do solo com potencial mátrico de $-1,5 \mathrm{MPa}$ (Richards, 1965); pelo teor de água do solo em que a resistência à penetração $\left(\theta_{\mathrm{RP}}\right)$ atinge $2 \mathrm{MPa}$ (Taylor et al., 1966); e pelo teor de água do solo em que a porosidade de aeração $\left(\theta_{\mathrm{PA}}\right)$ é de $10 \%$ (Grable \& Siemer, 1968).

No laboratório, as amostras foram devidamente preparadas e saturadas (Embrapa, 1997). Para obter a curva de retenção de água objetivando determinar o IHO, as amostras foram divididas em oito grupos de 16 unidades (2 profundidades x 2 manejos x 4 repetições). Cada grupo de amostras foi submetido à drenagem da água em uma das seguintes tensões: 0,$002 ; 0,004$; 0,$006 ; 0,01 ; 0,03 ; 0,1 ; 0,5 ;$ e $1,5 \mathrm{MPa}$. Para obter os potenciais mátricos $(\mathrm{Ym})$ de $-0,002,-0,004$ e $-0,006 \mathrm{MPa}$, utilizou-se mesa de tensão. Os $\psi \mathrm{m}$ de $-0,01,-0,033,-0,1,-0,5$ e - $1,5 \mathrm{MPa}$ foram obtidos por meio de pressões aplicadas às amostras colocadas sobre placas porosas em câmara de Richards (Klute, 1986).

Após atingirem o equilíbrio, as amostras foram pesadas e em seguida submetidas ao teste de resistência à penetração $(\mathrm{RP})$, utilizando o penetrógrafo eletrônico de bancada, da empresa Marconi, modelo MA 933, composto por uma célula de carga com capacidade nominal de $20 \mathrm{~kg}$ acoplada à extremidade de um braço mecânico, movimentado verticalmente 
por uma rosca-sem-fim. Uma haste metálica, com diâmetro de $6 \mathrm{~mm}$ e ponteira cônica com semiângulo de $30^{\circ}$ e área da base de $0,1256 \mathrm{~cm}^{2}$, foi utilizada para penetrar as amostras. A velocidade de deslocamento vertical da haste foi de $1,0 \mathrm{~cm} \mathrm{~min}^{-1}$ até a profundidade de $4,0 \mathrm{~cm}$. As medidas obtidas a partir da superfície da amostra até $1,0 \mathrm{~cm}$ de profundidade foram descartadas, conforme recomendado por Tormena et al. (1998). As leituras de RP foram obtidas por meio de um sistema automatizado de aquisição de dados e armazenadas em arquivos de extensão "txt". Logo após o teste de RP, as amostras foram secas em estufa a $105{ }^{\circ} \mathrm{C}$, por $48 \mathrm{~h}$, e pesadas novamente para determinação da umidade gravimétrica e da densidade do solo (Embrapa, 1997). A umidade volumétrica $(\theta \mathrm{v})$ foi obtida multiplicando-se a umidade gravimétrica pela densidade do solo (Klute, 1986).

A fim de obter os valores de $\theta \mathrm{CC}$ e $\theta \mathrm{PMP}$, utilizouse o modelo matemático referente à equação 1 , proposta por Silva et al. (1994), para ajuste dos dados originais, a qual incorpora a variável Ds na função empregada por Ross et al. (1991).

$$
\theta_{\mathrm{V}}=\exp (\mathrm{a}+\mathrm{bDs}) * \psi^{\mathrm{c}}
$$

em que $\theta_{\mathrm{V}}$ é a umidade volumétrica do solo $\left(\mathrm{cm}^{3} \mathrm{~cm}^{-3}\right)$; Ds é a densidade do solo $\left(\mathrm{g} \mathrm{cm}^{-3}\right) ; \psi$ é o potencial matricial (MPa); e a, b, c são coeficientes da equação.

Os valores de RP de todas as amostras com $\theta v$ e Ds conhecidas foram ajustados matematicamente, utilizando a equação 2, proposta por Busscher (1990). Por meio da relação funcional entre RP, $\theta v$ e Ds foi possível determinar o valor crítico de $\theta_{\mathrm{v}}$ para que a $\mathrm{RP}$ não ultrapassasse $2,0 \mathrm{MPa}\left(\theta_{\mathrm{RP}}\right)$, em função da Ds.

$$
R P=d \theta_{\mathrm{v}}{ }^{\mathrm{e}} * \mathrm{Ds}^{\mathrm{f}}
$$

em que RP é a resistência à penetração $(\mathrm{MPa}) ; \theta \mathrm{v}$ é a umidade volumétrica do solo $\left(\mathrm{cm}^{3} \mathrm{~cm}^{-3}\right)$; Ds é a densidade do solo $\left(\mathrm{g} \mathrm{cm}^{-3}\right)$; e d, e, f são coeficientes da equação.
Assim, para obter o valor de $\theta_{\mathrm{RP}}$, substitui-se RP, na equação 2 , pelo valor de 2,0 $\mathrm{MPa}$, considerado como limitante para efeito de cálculo do IHO.

Por fim, o valor de $\theta_{\mathrm{PA}}$ foi obtido por meio da equação 3 .

$$
\theta_{\mathrm{PA}}=(1-(\mathrm{Ds} / \mathrm{Dp}))-0,10
$$

em que $\theta_{\mathrm{PA}}$ é teor de água do solo em que a porosidade de aeração é igual a $10 \%\left(0,1 \mathrm{~cm}^{3} \mathrm{~cm}^{-3}\right)$; Ds é a densidade do solo $\left(\mathrm{g} \mathrm{cm}^{-3}\right)$; e Dp é a densidade real (densidade de partículas), assumindo-se o valor de $2,65 \mathrm{~g} \mathrm{~cm}^{-3}$ como valor da densidade média de partículas em solos argilosos.

Após o cálculo dos limites superiores e inferiores do IHO, determinou-se a densidade crítica do solo (Dsc), que é a densidade do solo em que o IHO se iguala a zero, ou seja, quando o limite superior do IHO equivale numericamente ao limite inferior (Silva et al., 1994).

Para o ajuste das equações 1 e 2 e obtenção dos coeficientes a, b, c, d, e, f, utilizou-se o programa estatístico SigmaPlot 8.0. Os limites inferiores e superiores do IHO em função da densidade do solo foram determinados por meio da planilha eletrônica do Excel ${ }^{\circledR}$, proposta por Leão \& Silva (2004). Os resultados de resistência à penetração e densidade do solo foram submetidos à análise de variância, e as médias, comparadas pelo teste t a $5 \%$.

\section{RESULTADOS E DISCUSSÃO}

Os intervalos hídricos ótimos (IHOs) obtidos para o sistema de manejo com MS estão apresentados na figura 1a,c, enquanto os IHOs do tratamento MBL apresentam-se na figura $1 \mathrm{~b}, \mathrm{~d}$, para as camadas de 0 a 20 e 20 a $40 \mathrm{~cm}$, respectivamente. Todas as curvas ajustadas, tanto de resistência à penetração como de retenção de água, apresentaram coeficientes de determinação significativos a $1 \%$ pelo teste $\mathrm{F}$ (Quadro 1).

Quadro 1. Ajuste das curvas de retenção de água no solo e de resistência à penetração de acordo com modelos não lineares propostos pela literatura, seguido dos coeficientes de determinação $\left(R^{2}\right)$, para os diferentes sistemas de manejo e profundidade de amostragem

Manejo Profundidade Equação da curva de resistência do solo e $\mathbf{R}^{2} \quad$ Equação da curva de resistência de água e $\mathbf{R}^{2}$

\begin{tabular}{lrlll}
\hline & $\mathrm{m}$ & & \\
$\mathrm{MS}$ & $0-20$ & $\mathrm{RP}=0.0006 \theta_{\mathrm{v}}^{-6.421} \mathrm{Ds}^{6.764} \mathrm{R}^{2}=0,89^{* *}$ & $\theta_{\mathrm{v}}=\exp (-2.0100+0.6100 \mathrm{Ds}) \psi^{-0.070} \mathrm{R}^{2}=0,85^{* *}$ \\
$\mathrm{MS}$ & $20-40$ & $\mathrm{RP}=0.0136 \theta_{\mathrm{v}}^{-3.274} \mathrm{Ds}^{9.383} \mathrm{R}^{2}=0.71^{* *}$ & $\theta_{\mathrm{v}}=\exp (-2.300+0.910 \mathrm{Ds}) \psi^{-0.050} \quad \mathrm{R}^{2}=0.83^{* *}$ \\
$\mathrm{MBL}$ & $0-20$ & $\mathrm{RP}=0.0041 \theta_{\mathrm{v}}^{-4.403} \mathrm{Ds}^{8.737} \mathrm{R}^{2}=0,90^{* *}$ & $\theta_{\mathrm{v}}=\exp (-2.880+1.310 \mathrm{Ds}) \psi^{-0.080}$ & $\mathrm{R}^{2}=0.88^{* *}$ \\
$\mathrm{MBL}$ & $20-40$ & $\mathrm{RP}=0.0056 \theta_{\mathrm{v}}^{-4.986} \mathrm{Ds}^{4.700} \mathrm{R}^{2}=0.90^{* *}$ & $\theta_{\mathrm{v}}=\exp (-2.170+0.760 \mathrm{Ds}) \psi^{-0.070}$ & $\mathrm{R}^{2}=0.86^{* *}$ \\
\hline
\end{tabular}

RP: resistência à penetração $(\mathrm{MPa})$; Ds: densidade do solo $\left(\mathrm{g} \mathrm{cm}^{-3}\right) ; \theta_{\mathrm{V}}$. umidade volumétrica do solo $\left(\mathrm{cm}^{3} \mathrm{~cm}^{-3}\right) ; \mathrm{Ds}_{\mathrm{s}}$ densidade do solo $\left(\mathrm{g} \mathrm{cm}^{-3}\right)$; $\psi$ : potencial matricial. MS: milho solteiro; MBL: milho consorciado com braquiária na linha de semeadura. ** significativo a $5 \%$ pelo teste $\mathrm{F}$. 
O IHO de ambas as camadas avaliadas (0 a 20 e 20 a $40 \mathrm{~cm}$ ) apresentou amplitude reduzida, pelo fato de o limite inferior de água no solo estar muito próximo ao limite superior. De acordo com Topp et al. (1994), é comum a ocorrência de valores muito baixos, ou até nulos, de IHO em solos de textura muito argilosa, o que, segundo Kay (1990), aumenta a probabilidade de ocorrência de limitações físicas para o crescimento das raízes no solo. Segundo Carter et al. (1999), o IHO em SSD é inferior ao encontrado em cultivos convencionais em decorrência de a $\mathrm{RP}$ atingir $2 \mathrm{MPa}$ em valores elevados de umidade, devido à elevada Ds encontrada no sistema conservacionista. Betz et al. (1998) e Cavalieri et al. (2006) também verificaram maiores valores do IHO em solo sob preparo convencional e escarificado, possibilitando um ambiente físico favorável ao crescimento das raízes.

Independentemente da camada avaliada, o IHO teve o teor de água para manter a $\mathrm{RP}$ em $2 \mathrm{MPa}\left(\theta_{\mathrm{RP}}\right)$ como limite inferior em $100 \%$ das amostras, superando a $\theta_{\mathrm{PMP}}$ em toda a amplitude de Ds encontrada. Esse resultado é típico de solos adensados, que necessitam estar com umidade elevada para que a RP não atinja o valor limitante, o que está de acordo com os relatos de Topp et al. (1994), Silva et al. (1994), Müller (2002) e Beutler et al. (2006), nos quais a RP foi o fator que mais reduziu o IHO em solos sob diferentes condições de textura e manejo, mesmo nos mais baixos valores de Ds. No entanto, em SSD com o cultivo de plantas de cobertura com sistema radicular agressivo e vigoroso, devido aos canais deixados com a morte dessas raízes, os quais orientam o crescimento radicular em profundidade da cultura sucessora (Silva \& Rosolem, 2001), a RP limitante para o crescimento das plantas pode ser superior à RP limitante em sistemas convencionais, em que a mobilização do solo causa a interrupção da continuidade dos macroporos e dos canais. Assim, se fosse considerada uma RP limitante superior a $2 \mathrm{MPa}$ para os manejos envolvendo o cultivo de plantas de cobertura, certamente aumentaria o IHO nesses tratamentos (Ehlers et al.,1983).

Nos dois sistemas de manejo (MS ou MBL) e nas duas camadas de solo avaliadas, o conteúdo de água disponível $\left(\mathrm{AD}=\theta_{\mathrm{CC}}-\theta_{\mathrm{PMP}}\right)$ foi sempre superior ao IHO, o que caracteriza os solos como fisicamente degradados (Letey, 1985). Por esse motivo, Silva et al. (1994) apontam o IHO como uma ferramenta de análise da estrutura do solo mais sensível que o conceito de água disponível.

Quanto ao limite superior do IHO, na camada de 0 a $20 \mathrm{~cm}$, a $\theta \mathrm{cc}$ foi o limite de água no solo em valores mais baixos de Ds, e a $\theta_{\mathrm{PA}}$ foi o limite superior em maiores valores de Ds. No tratamento com MS, a $\theta c c$ foi o limite máximo de água no solo até Ds de $1,28 \mathrm{~g} \mathrm{~cm}^{-3}$, quando $\theta_{\mathrm{PA}}$ assumiu o limite superior até a Ds de $1,35 \mathrm{~g} \mathrm{~cm}^{-3}$, valor em que o IHO passa a ser igual a zero. Já o tratamento MBL apresentou a $\theta c c$ como limite superior do IHO até a Ds de $1,27 \mathrm{~g} \mathrm{~cm}^{-3}$, e, a partir desse valor de $\mathrm{Ds}$, a $\theta_{\mathrm{PA}}$ foi o limite superior do IHO até a Ds de $1,30 \mathrm{~g} \mathrm{~cm}^{-3}$. Tormena et al. (1998) obtiveram resultados semelhantes em solo de textura muito argilosa, ou seja, a $\theta_{\mathrm{PA}}$ passou a ser o limite superior do IHO, substituindo a $\theta_{\mathrm{CC}}$, com a Ds de $1,28 \mathrm{~g} \mathrm{~cm}^{-3}$ e umidade volumétrica de $0,42 \mathrm{~cm} \mathrm{~cm}^{-3}$.

$\mathrm{Na}$ camada de 20 a $40 \mathrm{~cm}$, o sistema com MS (Figura 1c) apresentou $\theta_{\mathrm{CC}}$ como limite superior em $100 \%$ do IHO, ou seja, até a Ds de $1,23 \mathrm{~g} \mathrm{~cm}^{-3}$, valor esse considerado baixo para que o IHO se iguale a

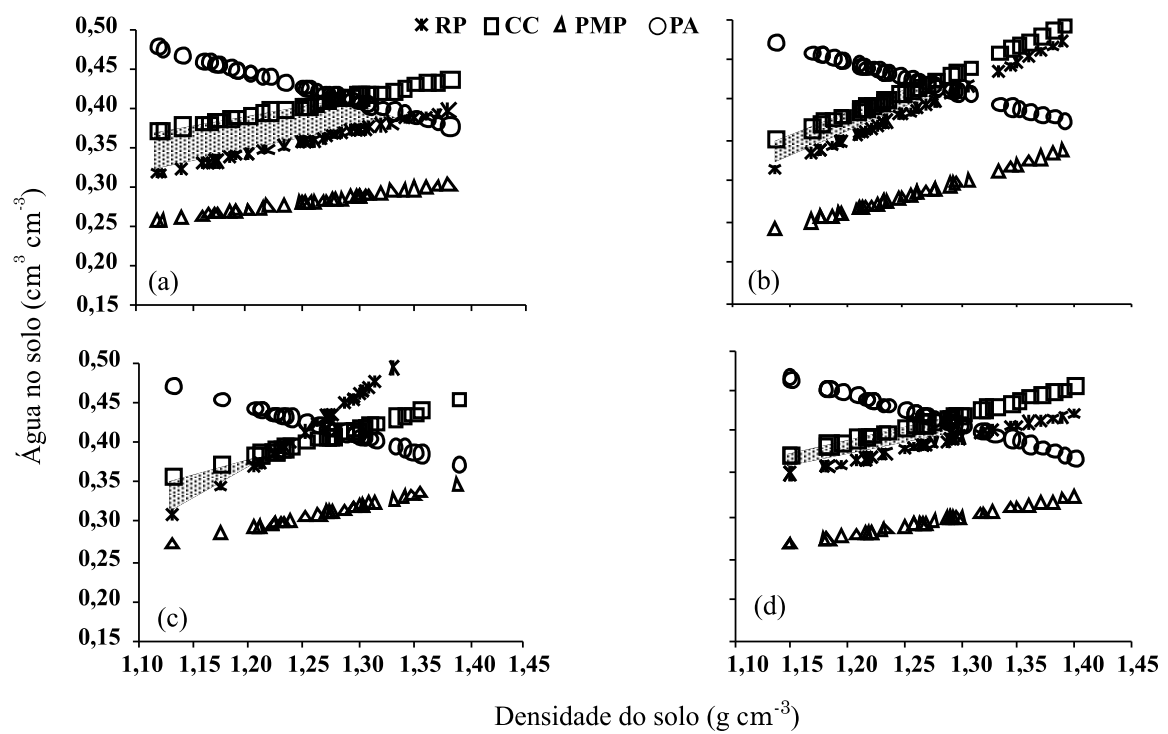

Figura 1. Variação do conteúdo de água no solo, em função da densidade aparente, para atender aos níveis críticos de capacidade de campo $(\mathrm{CC}=-0,01 \mathrm{MPa})$, ponto de murcha permanente $(\mathrm{PMP}=-1,5 \mathrm{MPa})$, porosidade de aeração $(\mathrm{PA}=10 \%)$ e resistência à penetração $(\mathrm{RP}=2 \mathrm{MPa})$. A área hachurada representa o IHO para os tratamentos milho solteiro $(a, c)$ e milho consorciado com braquiária na linha de semeadura (b, d), nas camadas de 0 a $20 \mathrm{~cm}(\mathrm{a}, \mathrm{b})$ e 20 a $40 \mathrm{~cm}(\mathrm{c}, \mathrm{d})$. 
zero (limite inferior igual ao limite superior de água no solo). Isso ocorreu devido à elevada compactação do solo nesse tratamento e nessa profundidade de amostragem, já que houve grande necessidade de aumentar o teor de água no solo para que a RP se mantivesse a $2 \mathrm{MPa}$ (limite inferior do IHO), atingindo a capacidade de campo (limite superior do IHO) em baixa densidade do solo. De acordo com Tormena et al. (1998), a RP apresenta maior impacto no IHO em camadas mais profundas do solo. Esse comportamento não foi verificado no tratamento MBL nessa camada de amostragem (Figura 1d), onde a $\theta_{\mathrm{CC}}$ foi o limite superior de água no solo até a Ds de $1,27 \mathrm{~g} \mathrm{~cm}^{-3}$, quando a $\theta_{\mathrm{PA}}$ passa a ser limitante até a Ds de $1,32 \mathrm{~g} \mathrm{~cm}^{-3}$, quando o IHO tornou-se nulo.

A Dsc, no conceito de IHO, é a densidade do solo em que o IHO iguala-se a zero, ou seja, em que o limite inferior de água no solo iguala-se ao limite superior (Silva et al., 1994); assim, quanto maior a Dsc, menor será a probabilidade de a planta permanecer em condições de estresse. Na camada de 0 a $20 \mathrm{~cm}$, o tratamento MS (Figura 2a) apresentou maior Dsc que o manejo com MBL (1,36 e 1,29 $\mathrm{g} \mathrm{cm}^{-3}$, respectivamente). Esses valores de Dsc são semelhantes ao encontrado por Müller (2002) em Nitossolo Vermelho com $590 \mathrm{~g} \mathrm{~cm}^{-3}$ de argila, na camada de 10 a $20 \mathrm{~cm}($ Dsc $=$ $1,33 \mathrm{~g} \mathrm{~cm}^{-3}$ ). Já Tormena et al. (1998) obtiveram Dsc de $1,28 \mathrm{~g} \mathrm{~cm}^{-3}$ em Latossolo Vermelho, com $800 \mathrm{~g} \mathrm{~cm}^{-3}$ de argila na camada de 0 a $10 \mathrm{~cm}$. Cavalieri et al. (2006) também observaram maior Dsc em tratamentos envolvendo solos manejados mecanicamente, reduzindo a probabilidade de ocorrência de amostras com densidades superiores à Dsc.

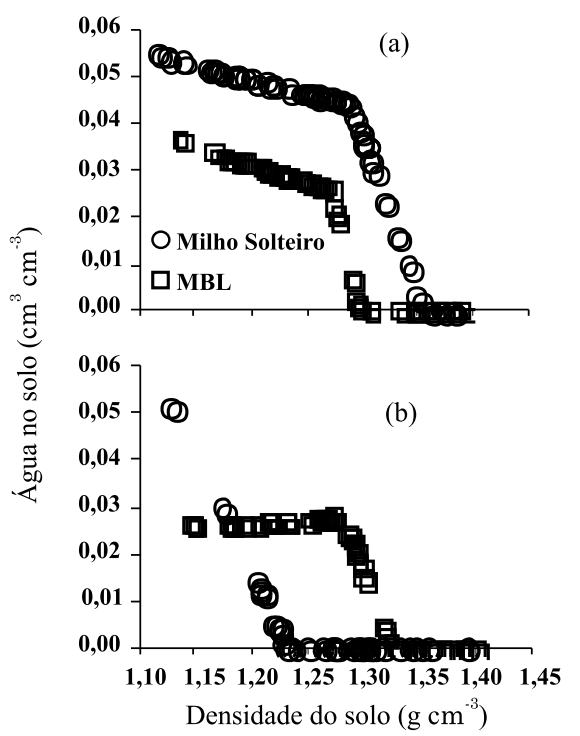

Figura 2. Variação do intervalo hídrico ótimo (IHO) em função da densidade do solo, nos sistemas de cultivo de milho solteiro (MS) e de milho consorciado com braquiária na linha de semeadura (MBL), nas profundidades de 0 $20 \mathrm{~cm}$ (a) e $20-40 \mathrm{~cm}$ (b).
O cultivo de braquiária em consórcio com o milho proporcionou, na camada de 20 a $40 \mathrm{~cm}$, uma Dsc $8 \%$ maior que a do cultivo de milho solteiro, ou seja, com valores de 1,33 e $1,23 \mathrm{~g} \mathrm{~cm}^{-3}$, respectivamente (Figura 2b). Calonego (2007) também observou aumento da Dsc com o cultivo de plantas de cobertura por três anos consecutivos em um Nitossolo Vermelho muito argiloso. Esse autor enfatiza ainda que o cultivo de plantas com sistema radicular agressivo e volumoso pode ser uma estratégia viável para melhoria da estrutura do solo a médio e longo prazo. Segundo Sharma \& Bhushan (2001), a adição de biomassa ao solo promoveu o aumento do IHO em função do aumento da $\theta_{\mathrm{PA}}$ e decréscimo da $\theta_{\mathrm{RP}}$, configurando um solo fisicamente melhor.

O efeito benéfico do cultivo de braquiária em consórcio com o milho na estruturação do solo em profundidade comprova-se pelos menores valores de RP (com umidade na capacidade de campo) encontrados na camada de 20 a $40 \mathrm{~cm}$ (Figura 3). Assim, o cultivo de braquiária semeada em consórcio com o milho na linha de semeadura promoveu maiores efeitos na estruturação do solo em profundidade, com melhorias nas qualidades físicas e físico-hídricas, provavelmente em decorrência do grande aporte de matéria seca radicular no perfil do solo por essa forrageira perene. A ausência de efeito do cultivo consorciado da forrageira com milho na estruturação do solo em camadas mais superficiais, como de 0 a $20 \mathrm{~cm}$, deve-se, provavelmente, à grande densidade das raízes de milho nessa profundidade, sendo suficiente para a estruturação superficial do solo. Silva et al. (2000), ao estudarem a distribuição do sistema radicular de milho na camada de 0 a $40 \mathrm{~cm}$, verificaram que $83 \%$ do total de raízes encontrava-se na camada de 0 a $20 \mathrm{~cm}$.

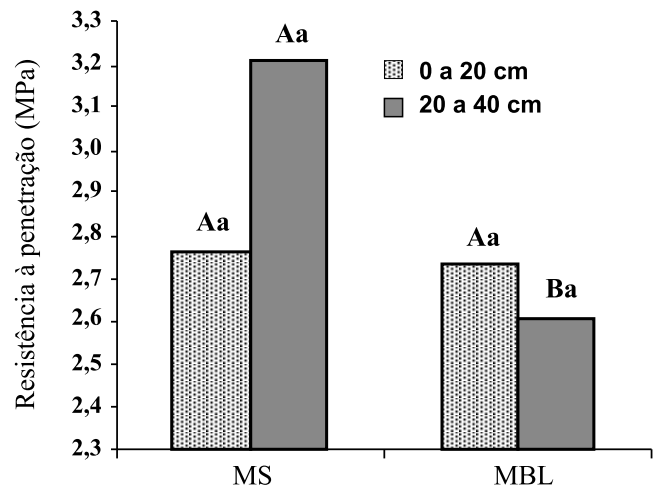

Figura 3. Resistência mecânica à penetração com umidade na capacidade de campo, nos sistemas de manejo de milho solteiro (MS) e de milho consorciado com braquiária na linha de semeadura (MBL). Letras iguais não diferem entre si a $5 \%$ pelo teste t. Letras maiúsculas comparam os sistemas de cultivo dentro de cada profundidade, e letras minúsculas comparam as profundidades dentro de cada sistema de cultivo. 
O cultivo por dois anos de milho consorciado com braquiária melhorou a estruturação do solo na camada de 20 a $40 \mathrm{~cm}$ de profundidade em relação ao cultivo de milho solteiro, contrariando resultados obtidos por Denardin et al. (2008), em que os atributos físicos e químicos do solo não foram influenciados pelo cultivo da braquiária em consórcio com milho.

Segundo Denardin et al. (2001), a frequência, a quantidade e a qualidade do material orgânico adicionado ao solo resultam em sua melhoria estrutural. Nesse sentido, no "Sistema Santa Fé" a braquiária constitui opção de destaque entre as espécies com potencial para promover melhorias à estrutura do solo, em razão da quantidade, qualidade e distribuição de fitomassa radicular que adiciona ao solo (Kluthcouski et al., 2004; Fonseca et al., 2007; Crusciol et al., 2010), podendo reverter situações de compactação do solo devido à satisfatória tolerância à resistência à penetração dessa espécie forrageira (Silva, 2004).

\section{AGRADECIMENTOS}

À FAPESP pelo financiamento da pesquisa e ao CNPq pela bolsa de produtividade em pesquisa concedida ao terceiro autor.

\section{CONCLUSÕES}

1. O cultivo solteiro de milho é suficiente para melhorar as características físicas e estruturais do solo na camada de 0 a $20 \mathrm{~cm}$.

2. O cultivo de braquiária em consórcio com o milho por dois anos consecutivos melhora as condições físicas e estruturais do solo na camada de 20 a $40 \mathrm{~cm}$, em relação ao milho solteiro, com redução da resistência mecânica à penetração e aumento da densidade crítica do solo no conceito de intervalo hídrico ótimo.

\section{LITERATURA CITADA}

ANDRADE, R.S.; STONE, L.F. \& SILVEIRA, P.M. Culturas de cobertura e qualidade física de um Latossolo em plantio direto. R. Bras. Eng. Agríc. Amb., 13:411-418, 2009.

BETZ, D.L.; ALLMARAS, R.R.; COPELAND, S.M. \& RANDALL, G.W. Least limiting water range: Traffic and long-term tillage influences in a Webster soil. Soil Sci. Soc. Am. J., 62:1384-1393, 1998 .

BEUTLER, A.N.; CENTURION, J.F.; SILVA, A.P. \& BARBOSA, J.C. Intervalo hídrico ótimo e produtividade de cultivares de soja. R. Bras. Eng. Agr. Amb., 10:639$645,2006$.
BORGHI, E. \& CRUSCIOL, C.A.C. Produtividade de milho, espaçamento e modalidade de consorciação com Brachiaria brizantha no sistema plantio direto. Pesq. Agropec. Bras, 42:163-171, 2007.

BUSSCHER, W.J. Adjustment of that-tipped penetrometer resistance data to a common water content. Trans. ASAE, 33:519-524, 1990

CALONEGO, J.C. Uso de plantas de cobertura na recuperação de solo compactado. Botucatu, Universidade Estadual Paulista, 2007. 125p. (Tese de Doutorado)

CALONEGO, J.C. \& ROSOLEM, C.A. Estabilidade de agregados do solo após manejo com rotações de culturas e escarificação. R. Bras. Ci. Solo, 32:1399-1407, 2008.

CARTER, M.R.; ANGERS, D.A. \& TOPP, G.C. Characterizing equilibrium physical condition near the surface of a fine sandy loam under conservation tillage in a humid climate. Soil Sci., 164:101-110, 1999.

CAVALIERI, K.M.V.; TORMENA, C.A.; VIDIGAL FILHO, P.S.; GONÇALVES, A.C.A. \& COSTA, A.C.S. Efeitos de sistemas de preparo nas propriedades físicas de um Latossolo Vermelho distrófico. R. Bras. Ci. Solo, 30:137147,2006

CRUSCIOL, C.A.C.; SORATTO, R.P.; BORGHI, E. \& MATEUS, G.P. Integração Lavoura-Pecuária: Benefícios das gramíneas perenes no sistema de produção. Piracicaba, Potafós, 2009. 14p. (Informações Agronômicas, 125)

CRUSCIOL, C.A.C.; SORATTO, R.P.; BORGHI, E. \& MATEUS, G.P. Benefits of integrating crops and tropical pastures as systems of production. Better Crops, 94:14-16, 2010.

DENARDIN, J.E.; KOCHHANN, R.A. \& DENARDIN, N.D. Considerações sobre adensamento e compactação em manejo de Latossolos. In: WORKSHOP COESÃO EM SOLOS DOS TABULEIROS COSTEIROS, Aracajú, 2001. Anais... Aracajú, Embrapa Tabuleiros Costeiros, 2001. p.317-325.

DENARDIN, J.E.; KOCHHANN, R.A.; SANTI, A.; FAGANELLO, A. \& SATTLER, A. Efeito da consorciação milho-braquiária (Brachiaria brizantha) na mitigação da compactação do solo. Passo Fundo, Embrapa Trigo, 2008. 13p. html (Embrapa Trigo. Boletim de Pesquisa e Desenvolvimento Online, 54). Disponível em: <http:// www.cnpt.embrapa.br/biblio/bp/p_bp54.htm>. Acesso em: 4 de maio de 2011

DIAS JÚNIOR, M.S. Compactação do solo. In: NOVAIS, R.F.; ALVAREZ V., H.V. \& SCHAEFER, C.E.G.R., ed. Tópicos em ciência do solo. Viçosa, MG, Sociedade Brasielira de Ciência do Solo, 2000. v.1. p.55-94.

EHLERS, W.; KOPKE, V.; HESSE, F. \& BOHM, W. Penetration resistance and root growth of oats tilled and untilled loess soil. Soil Tillage Res., 3:261-275, 1983.

EMPRESA BRASILEIRA DE PESQUISA AGROPECUÁRIA EMBRAPA. Centro Nacional de Pesquisa de Solos. Sistema brasileiro de classificação de solos. 2.ed. Rio de Janeiro, 2006. 306p. 
EMPRESA BRASILEIRA DE PESQUISA AGROPECUÁRIA EMBRAPA. Centro Nacional de Pesquisa de Solos. Manual de métodos de análises de solos. 2.ed. Rio de Janeiro, 1997. 212p.

FONSECA, G.C.; CARNEIRO, M.A.; COSTA, A.R.; OLIVEIRA, G.C. \& BALBINO, L.C. Atributos físicos, químicos e biológicos de Latossolo Vermelho distrófico de Cerrado sob duas rotações de cultura. Pesq. Agropec. Trop., 37:22 30, 2007.

GRABLE, A.R. \& SIEMER, E.G. Effects of bulk desnsity, aggregate size, and soil water suction on oxygen diffusion, redox potencial and elongation of corns roots. Soil Sci. Soc. Am. J., 32:180-186, 1968.

HAISE, H.R.; HASS, H.J. \& JENSEN, L.R. Soil moisture studies of some great plains soils. II. Field capacity as related to $1 / 3$ atmosphere percentege, and "minimum point"as related to 15- and 26- atmosphere percentege. Soil Sci. Soc. Am. Proc., 34:20-25, 1955

JAKELAITIS, A.; SILVA, A.F.; SILVA, A.A.; FERREIRA, L.R.; FREITAS, F.C.L. \& VIANA, R.G. Influência de herbicidas e de sistemas de semeadura de Brachiaria brizantha consorciada com milho. Planta Daninha, 23:59-68, 2005.

KAY, B.D. Rates of changes of soil structure under different cropping systems. Adv. Soil Sci., 12:1-51, 1990.

KLUTE, A. Water retention: Laboratory methods. In: KLUTE, A., ed. Methods of soil analysis. 2.ed. Madison, American Society of Agronomy, 1986. Part. 1. p.635-662.

KLUTHCOUSKI, J.; COBUCCI, T.; AIDAR, H.; YOKOYAMA, L.P.; OLIVEIRA, I.P.; COSTA, J.L.S.; SILVA, J.G.; VILELA, L.; BACELLOS, A.O. \& MAGNABOSCO, C.U. Sistema Santa Fé - Tecnologia Embrapa: Integração lavoura-pecuária pelo consórcio de culturas anuais com forrageiras, em áreas de lavoura, nos sistemas direto e convencional. Santo Antonio de Goiás, Embrapa Arroz e Feijão, 2000. 28 p. (Circular Técnica, 38)

KLUTHCOUSKI, J.; AIDAR, H.; STONE, L.F. \& COBUCCI, T. Integração lavoura-pecuária e o manejo de plantas daninhas. Piracicaba, Potafós, 2004. 20p. (Informações Agronômicas, 106)

LEÃO, T.P. \& SILVA, A.P. A simmplified excel algarithm for estimating the least limiting water range of soils. Sci. Agric., 61:649-654, 2004.

LETEY, J. Relationship between soil physical properties and crop production. Adv. Soil Sci., 1:277-294, 1985.

LOMBARDI NETO, F. \& DRUGOWICH, M.I. Manual técnico de manejo e conservação de solo e água. Campinas, CATI, 1994. v.2. 168p.

MÜLLER, M.M.L. Influência do monocultivo na cana-de-açúcar e nas propriedades físicas e químicas de um Nitossolo Vermelho e um Neossolo Quartzarênico. Botucatu, Universidade Estadual Paulista, 2002. 120p. (Tese de Doutorado)
RAIJ, B.van; CANTARELLA, H.; QUAGGIO, J.A. \& FURLANI, A.M.C. Recomendações de adubação e calagem para o Estado de São Paulo. 2.ed. Campinas, Instituto Agronômico de Campinas, 1996. 285p.

RICHARDS, L.A. Physical conditions of water in soil. In: BLACK, C.A.; EVANS, D.D.; WHITE, J.L.; ENSMINGE, L.E. \& CLARK, F.E., ed. Methods of soil analysis - Physical and mineralogical properties, including statistics of measurements and sampling. Madison, ASA/SSSA, 1965. p.128-152

ROSS, P.J.; WILLIANS, J. \& BRISTOW, K.L. Equations for extending water-retention curves to drynees. Soil Sci. Soc. Am. J., 55:923-927, 1991.

SHARMA, P.K. \& BHUSHAN, L. Physical characterization of a soil amended with organic residues in a rice-wheat cropping system using a single value soil physical index. Soil Tillage Res., 60:143-152, 2001.

SILVA, A.P.; KAY, B.D. \& PERFECT, E. Characterization of the least limiting water range. Soil Soil Sci. Soc. Am. J., 58:1775-1781, 1994.

SILVA, G.J. Desenvolvimento de plantas de soja, milho, algodão e Brachiaria brizantha, submetidas a quatro graus de compactação de um Latossolo Vermelho-Escuro distrófico. Cuiabá, Universidade Federal do Mato Grosso, 2004. 122p. (Tese de Mestrado)

SILVA, V.R.; REINERT, D.J. \& REICHERT, J.M. Densidade do solo, atributos químicos e sistema radicular do milho afetados pelo pastejo e manejo do solo. R. Bras. Ci. Solo, 24:191-199, 2000.

SILVA, R.H. \& ROSOLEM, C.A. Crescimento radicular de espécies utilizadas como cobertura decorrente da compactação do solo. R. Bras. Ci. Solo, 25:253-260, 2001.

TAYLOR, H.M.; ROBERSON, G.M. \& PARKER Jr., J.J. Soil strength-root penetration relations to medium to coarsetextured soil materials. Soil Sci., 102:18-22, 1966.

TAYLOR, H.M. Root behavior as affected by soil structure and strength. In: CARSON, E.W. The plant root and its environment. Charlottesville, University Press of Virginia, 1974. p.271-291.

TOPP, G.C.; GALGANOV, Y.T.; WIRES, K.C. \& CULLEY, J.L.B. Non limiting water range (NLWR): An approach for assessing soil structure. Ottawa, Soil Quality Evaluation Program/Agriculture and Agr-Food Canada, 1994. 36p. (Technical Report, 2)

TORMENA, C.A.; SILVA, A.P. \& LIBARDI, P. Caracterização do intervalo hídrico ótimo de um Latossolo Roxo sob plantio direto. R. Bras. Ci. Solo, 22:573-581, 1998.

ZANINE, A.M.; SANTOS, E.M.; FERREIRA, D.J. \& CARVALHO, G.G.P. Potencialidade da integração lavourapecuária: Relação planta-animal. R. Eletrónica Vet., 7, 2006. Disponível em: <http://www.veterinaria.org/ revistas/redvet/n010106/010601.pdf > Acesso em: 04 de maio de 2011 